\title{
Mikroresonatorarray: Ein besonderer optischer Sensor
}

\author{
Thomas Weigel, Gustav Schweiger, Cemal Esen, Andreas Ostendorf \\ Laseranwendungstechnik, Ruhr-Universität Bochum, Universitätsstr. 150, 44801 Bochum, \\ Deutschland
}

\section{Zusammenfassung}

Sphärische Mikroresonatoren stellen eine hochsensitive Messmethode dar. Bisher durchgeführte Messungen an einzelnen Partikeln sind jedoch aufwendig und stellen hohe Ansprüche an das Messsystem, was eine Anwendung erschwert. Im Rahmen dieses Artikels wird ein neuartiges Verfahren vorgeschlagen, welche diese Probleme deutlich reduziert und somit einen Zugang zu einer technologischen Umsetzung sphärischer optischer Mikroresonatoren als Sensor bietet.

Keywords: optische Sensoren, Resonatoren, Mikrokugeln

\section{Einleitung}

Optische Sensoren zeichnen sich zumeist durch ihre hohe Sensitivität, Immunität gegen elektromagnetische Felder und sehr kleine Baugröße aus. Dies trifft in besonderem Maße auf faseroptischen Messverfahren zu, die auf optischen Interferenzeffekten beruhen. Diese werden für eine Vielzahl unterschiedlicher physikalischer Größen wie Temperatur, Druck oder auch zur Detektion von chemischen oder biologischen Substanzen eingesetzt. Eine Übersicht über faserbasierte Sensoren ist z.B. dem Werk von Udd und Spillman [Udd, Spillman 2011, 1] zu entnehmen. Bei all diesen Verfahren wird das spektrale Verhalten durch die Messgröße verändert. Dies zeigt sich etwa in der Verschiebung von Transmissions- oder Resonanzspitzen. Die Sensitivität des Messsystems lässt sich durch die Gütekennzahl $Q$ charakterisieren, welche sich im Falle eines Resonators aus dem Verhältnis zwischen gespeicherter Energie und Verlusten pro Umlauf einer Welle im Resonator ergibt. Sie ist entscheidend von der Geometrie des verwendeten Resonators abhängig. Kugelförmige Resonatoren zeichnen sich aufgrund ihrer hohen Symmetrie durch besonders hohe Gütekennzahlen aus, die es möglich machen selbst einzelne Moleküle nachzuweisen [2-4].

\section{Einführung in Whispering-Gallery Mo- des}

Der Name Whispering Gallery Modes, WGM (deutsch: Flüstergalleriemoden) gehen auf Untersuchungen von Lord Rayleigh an Schallwellen in der Kuppel der St. Paul Kathedrale in London in der zweiten Hälfte des 19. Jahrhunderts zurück. Das zugrundeliegende Phänomen ist auch für optische Resonanzen an die-

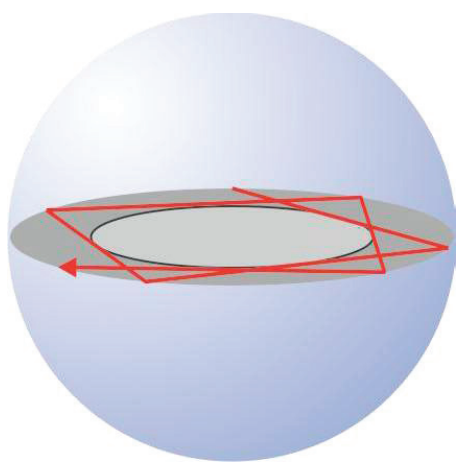

Abb. 1: Entstehung von Whispering Gallery Moden in kugelförmigen Resonatoren

lektrischen Kugeln gültig. Eine Prinzipskizze ist in Abb. 1 dargestellt. Obwohl die Wechselwirkung des Lichtfelds mit dem Partikel mit Hilfe der Wellenoptik exakt beschrieben werden kann, wollen wir uns einer Beschreibung mittels geometrischer Optik bedienen, da dies einen besseren und intuitiveren Einblick in die physikalischen Zusammenhänge bietet. In der geometrischen Optik wird das Lichtfeld durch eine Anzahl von Strahlen unter Berücksichtigung ihrer Phase beschrieben. Läuft einer dieser Strahlen im Partikel, wie in Abb. 1 gezeigt, um, so kommt es an den Schnittpunkten mit sich selbst zu Interferenzen. Unter bestimmten Umständen kommt es an allen Schnittpunkten zu einer konstruktiven Überlagerung und somit zu einer resonant umlaufenden Welle. Aufgrund der Symmetrie des Systems kann der Strahl innerhalb des Partikels die Einfallsebene nicht verlassen, sodass sich das Problem zweidimensional beschreiben 


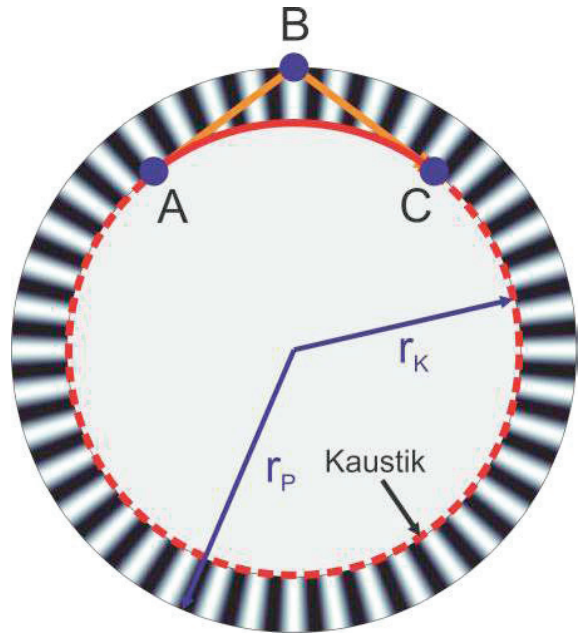

Abb. 2: Illustration der Resonanzbedingung für einen kugelförmigen Resonator

lässt.

Im Bild der geometrischen Optik ergibt sich, wie in Abb. 2 skizziert, genau dann eine Resonanz, wenn der Phasenfortschritt des Strahls $\Delta \phi_{S}$ entsprechend entlang der Strecke von $A$ über B nach $\mathrm{C}$ dem Phasenfortschritt $\Delta \phi_{K}$ entlang der Kaustik bis auf ein Vielfaches von $2 \pi$ entspricht:

$$
\begin{aligned}
& \Delta \phi_{S}=n_{P} k_{0} 2 \sqrt{r_{P}^{2}-r_{K}^{2}}-\frac{\pi}{2}-\delta \phi_{\text {reff }} \\
& \Delta \phi_{K}=n_{P} k_{0} 2 r_{K} \arccos \frac{r_{K}}{r_{P}} \\
& \Delta \phi_{S}-\Delta \phi_{K}=(m-1) \cdot 2 \pi
\end{aligned}
$$

Hierbei bezeichnen $r_{P}$ und $r_{K}$ die Radien des Partikels bzw. der Kaustik, $n_{P}$ den Brechungsindex des Partikels, $k_{0}=2 \pi / \lambda_{0}$ die Wellenzahl im Vakuum, $\delta \phi_{\text {reff. }}$ den Phasensprung bei Reflexion an der Partikeloberfläche, sowie $m$ die Resonanzordnung. Der Faktor $\pi / 2$ lässt sich durch die Eigenfokussierung des Strahls an der Kaustik erklären (näheres hierzu z.B. in [van Hulst 1981, 5]). Die Anwendung optischer Resonatoren für die Sensorik lässt sich aus den Einflussgrößen in den Gleichungen (1a)(1c) erkennen. Direkt ist die Abhängigkeit von der Größe des Partikels und von dessen Brechungsindex zu erkennen. Prinzipiell lassen sich Resonanzen auch mit Strahlen erzeugen, die durch Brechung in das Partikel gelangen, jedoch sind solche Resonanzen sehr stark verlustbehaftet und somit deutlich verbreitert. Scharfe Resonanzen lassen sich daher nur durch Strahlen erreichen, die an der Grenzfläche totalreflektiert werden. In diesem Fall ist der Phasensprung [6] bei Reflexion an der Oberfläche ebenfalls von dem Brechungsindex

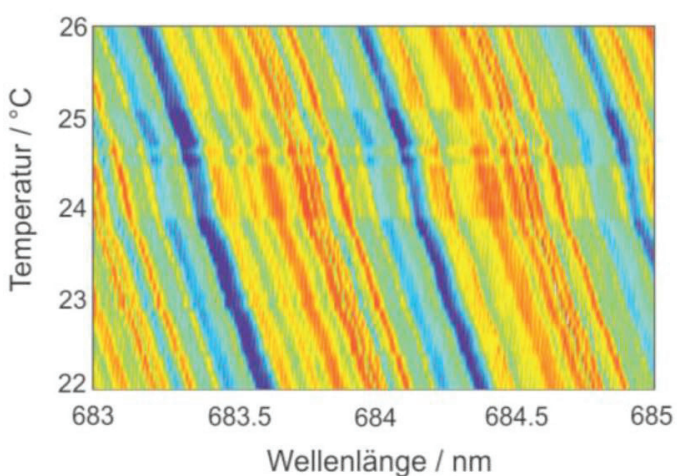

Abb. 3: Resonanzspektrum eines PMMAPartikels bei schrittweise Änderung der Umgebungstemperatur. Die Amplituden der Spektren sind farbkodiert.

der Umgebung abhängig. Eine weitere Einflussgröße stellt die Temperatur der Umgebung dar, da diese einen Einfluss sowohl auf die Größe des Partikels als auch dessen Brechungsindex hat. Dies bewirkt eine Verschiebung der Resonanzen. Beispielhaft ist in Abb. 3 der Einfluss der Temperatur auf das Resonanzverhalten eines Partikels aus Polymethylmetacrylat (PMMA) dargestellt. Hier wurde die Temperatur schrittweise im Bereich zwischen 22 und $26^{\circ} \mathrm{C}$ verändert. Gleichzeitig wurde das Resonanzspektrum aufgezeichnet. Es ist deutlich der lineare Zusammenhang zwischen resonanter Wellenlänge und Temperatur zu erkennen. Die Verschiebung ist stark materialabhängig und beträgt z.B. bei PMMA etwa $100 \mathrm{pm} /{ }^{\circ} \mathrm{C}$, ist jedoch unabhängig von der Größe des Partikels, wie dies in Abb. 4 zu sehen ist. Zur Bestimmung der Verschiebung der resonanten Wellenlänge wurde eine durchstimmbare Laserquelle (NewFocus Velocity 6308) mit einer minimalen Scangeschwindigkeit von ca. $0,1 \mathrm{~nm} / \mathrm{s}$ verwendet. Zur Detektion wurde eine CMOS-Camera (VRMagic 3+pro) mit einer Bildwiederholrate von ca. 80 Bilder/s eingesetzt. Dies ergibt eine

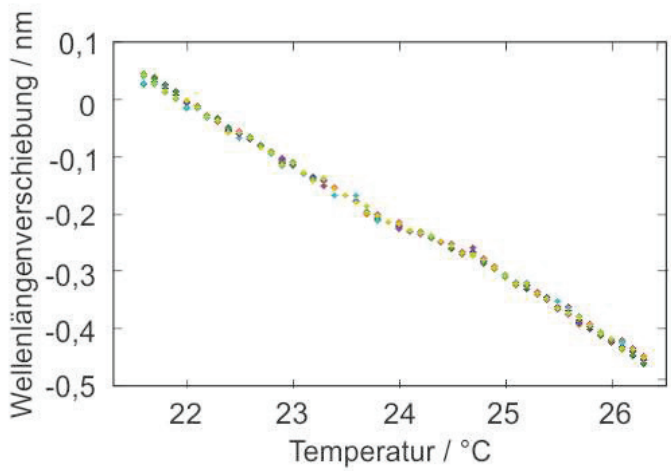

Abb. 4: Resonanzverschiebung von 13 unterschiedlichen Mikroresonatoren als Funktion der Temperatur 
theoretische Empfindlichkeit von 1,25pm, was einer Temperaturempfindlichkeit von ca. $0,01^{\circ} \mathrm{C}$ entspricht. Mit einem solchen optischen Mikroresonator lässt sich die Temperatur genau bestimmen. Jedoch wird hierfür ein kostspieliges, präzise durchstimmbares Lasersystem benötigt. Dieses Problem lässt sich durch die Verwendung von mehreren Mikroresonatoren leicht unterschiedlicher Größe umgehen.

\section{Mikropartikelarray als optischer Sensor}

Das Prinzip des Mikropartikelarrays als Sensor soll hier am Beispiel der Wellenlängenbestimmung näher beschrieben werden. Entsprechend Abb. 5 wird ein Prisma derart beleuchtet, dass es zu einer Totalreflexion an der Unterseite der Messzelle (Grundfläche des Prismas) kommt. Die einfallende Strahlung kann im Wesentlichen durch eine evaneszente Kopplung in die Mikropartikel geleitet werden. Dies erhöht den Kontrast deutlich, da bei einer refraktiven Kopplung das Streulicht die resonante Strahlung überdecken würde. Die Messzelle besteht aus einem Substrat auf dem sich die Mikroresonatoren befinden und einem Fenster aus Glas. Zur Detektion wird, wie bereits zuvor erwähnt, eine einfache CMOSKamera eingesetzt. Die Weiterverarbeitung der aufgenommen Bilder erfolgt über eine entsprechende Auswertesoftware.

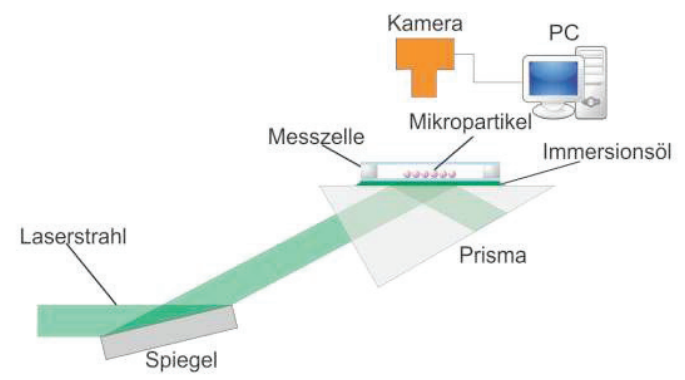

Abb. 6: Experimenteller Aufbau

Als optische Resonatoren wurden Mikropartikel aus PMMA der Fa. MicroParticles, Berlin und sowie Kalknatronglaspartikel der Fa. Cospheric im Größenbereich von 90-120um eingesetzt. Die Mikropartikel besitzen leicht unterschiedliche Größen. Dies führt zu einer Variation des Resonanzverhaltens der Partikel. Bei einer bestimmten Wellenlänge ergibt sich eine spezifische Intensitätsverteilung der Partikel,
Aufnahme einer Spektrum eines Intensitätsverteilung Mikropartikels

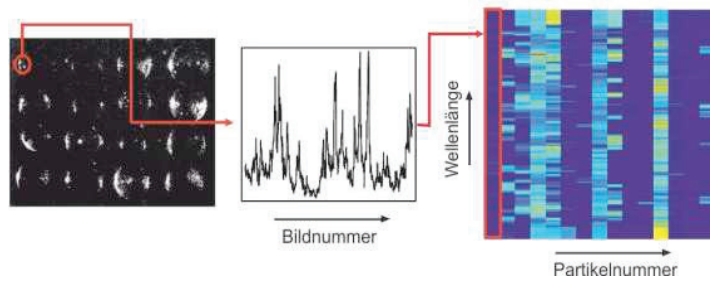

Abb. 5: Erstellung einer Referenzdatenbank aus Intensitätsaufnahmen während eines Wellenscans der Referenzlaserquelle

welche als Fingerabdruck für die Wellenlänge verwendet werden kann. Wie in Abb. 6 dargestellt, wird in einem Kalibrierungsvorgang die Wellenlänge einer durchstimmbaren Laserquelle kontinuierlich verändert. Gleichzeitig werden mittels einer einfachen CMOS-Kamera Aufnahmen des Mikropartikelarrays gemacht (Abb. 6 links). Für jedes Partikel werden die Intensitäten aus den Kameraaufnahmen gewonnen. Beispielhaft ist das Spektrum einer Kugel in Abb. 6 (Mitte) dargestellt. Jedes Bild ist dabei über den Zeitpunkt der Aufnahme und der Scangeschwindigkeit des Lasers eindeutig mit einer bestimmten Wellenlänge verknüpft. Die Spektren der einzelnen Kugel werden dann wie in Abb. 6 (rechts) dargestellt in einer Datenbank zusammengefasst. Will man nun die Wellenlänge einer unbekannten Laserquelle bestimmen, so vergleicht man einfach die entsprechende Intensitätsverteilung mit der Datenbank. Durch einen direkten Vergleich lässt sich dann die unbekannte Wellenlänge bestimmen. Dieser Vergleich erfolgt über eine Vergleichsfunktion $V(\lambda)$ :

$V(\lambda)=\sum_{j}\left(I_{j}^{D B}(\lambda)-I_{j}\left(\lambda_{u}\right)\right)^{2}$

Hierbei wird eine Summation über alle Kugeln $j$ im Array durchgeführt. $I_{j}\left(\lambda_{u}\right)$ stellt dabei die Intensität der $j$-ten Kugel bei der unbekannten Wellenlänge dar und $I_{j}^{P B}(\lambda)$ die Intensität der entsprechenden Kugel in der Datenbank. Mit Hilfe der Vergleichsfunktion können prinzipiell auch mehrere Wellenlängen identifiziert werden. Eine Begrenzung stellt hier lediglich die spektrale Breite der Lichtquellen dar. Diese sollte nicht größer 10-20pm) sein. Eine Messung mit zwei Laserquellen mit leicht unterschiedlichen Wellen ist in Abb. 7 dargestellt. Die Wellenlängen können über die Minima der Vergleichsfunktion identifiziert werden. 


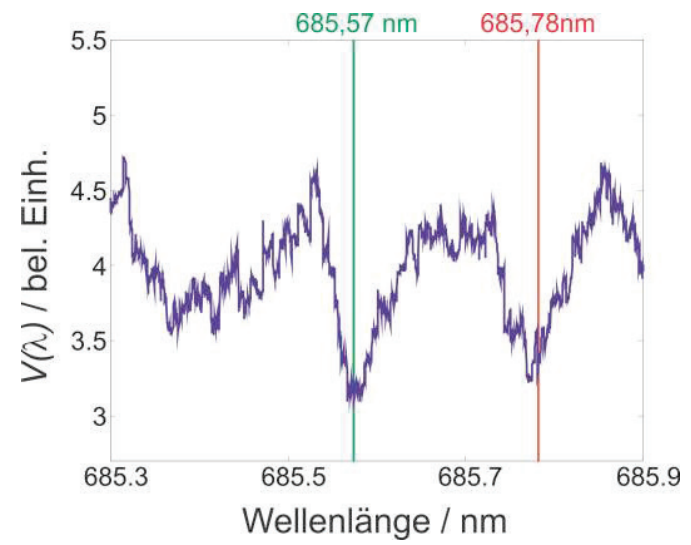

Abb. 7: Identifikation zweier Laser mit leicht unterschiedlichen Wellenlängen mit Hilfe der Vergleichsfunktion $V(\lambda)$

\section{Wellenlängenbestimmung}

Für die Bestimmung der Wellenlänge einer unbekannten Laserquelle wird zunächst eine Datenbank angelegt wie, dies zuvor beschrieben wurde. Einen Vergleich mit einem kommerziell erhältlichen Wavemeter (EXFO WA1500), mit einer Messgenauigkeit von 0,2pm ist in Abb. 8 dargestellt.Dabei ist eine sehr gute Übereinstimmung mit der Referenzmes-

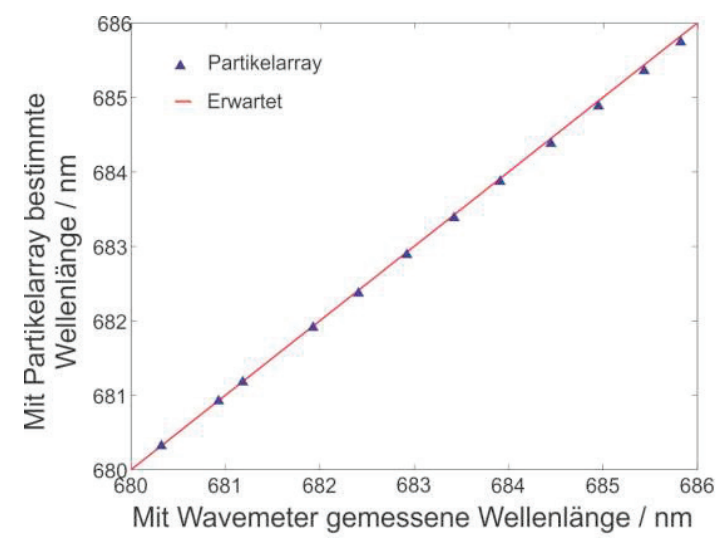

Abb. 9: Vergleich der Wellenlängenbestimmung durch das Mikropartikelarray mit einer Messung durch ein Wavemeter

sung zu erkennen. Der Messbereich ist hier durch den Durchstimmbereich des während des Kalibrierungsvorgangs verwendeten Lasersystems bestimmt. Jedoch kann das beschriebene Messverfahren aufgrund der erreichbaren Genauigkeit z.B. für die Überwachung von Lasersystemen verwendet werden, um ein Abdriften der Wellenlänge, etwa aufgrund von Temperatureffekten, frühzeitig erkennen zu können. Das zur Kalibrierung notwendige kostspielige, durchstimmbare Lasersystem lässt sich durch ein gezieltes thermisches Durchstimmen ersetzen. Bei Änderung der Temperatur des Resonators verändert sich neben der Größe des Partikels durch thermische Ausdehnung auch der Brechungsindex, dies führt gemäß [7] zu einer Verschiebung der Resonanzfrequenz:

$$
\frac{1}{\lambda} \frac{d \lambda}{d T}=\frac{1}{r} \frac{d r}{d T}+\frac{1}{n} \frac{d n}{d T}=\left(\alpha_{T}+\alpha_{n}\right) \cdot \Delta T
$$

Mit dem Wärmeausdehnungskoeffizienten $\alpha_{T}$ und dem thermo-optischen Koeffizienten $\alpha_{n}$.

Tab. 1: Thermische Eigenschaften von PMMA und Glas

\begin{tabular}{c|l|l} 
Material & $\alpha_{T} / \mathrm{K}^{-1}$ & $\alpha_{n} / \mathrm{K}^{-1}$ \\
\hline PMMA & $52-84 \cdot 10^{-6}[8 ; 9]$ & $-1,10^{-4}[10]$ \\
\hline \multirow{3}{*}{ Glas } & $\begin{array}{l}5,5-13 \cdot 10^{-6}[11] \\
\text { (abh. von der } \\
\text { Glassorte) }\end{array}$ & $\begin{array}{l}8,86 \cdot 10^{-6} \\
{[12]} \\
\text { (Quartzglas) }\end{array}$
\end{tabular}

Wie aus Tab. 1 zu entnehmen ist, hat PMMA gegenüber Glas einen um zwei Größenordnungen höheren thermo-optischen Koeffizienten. Dies führt zu einer experimentell bestimmten thermischen Verschiebung der Resonanzwellenlänge von $\sim 100 \mathrm{pm} /{ }^{\circ} \mathrm{C}$, gegenüber $\sim 5 \mathrm{pm} /{ }^{\circ} \mathrm{C}$ bei Glas. Dadurch ist der thermische Scanbereich bei PMMA deutlich höher, jedoch stellt die Aufnahme von Wasser aus der Atmosphäre ein Problem dar (vgl. etwa [13]), da diese deutlich von der Temperatur abhängt. Aus diesem Grund wurde für den Vergleich von Temperaturscan und Wellenlängenscan Glaspartikel verwendet.Die Ergebnisse sind beispielhaft für vier unterschiedliche Partikel in Abb. 9 dargestellt. Zur besseren Vergleichbarkeit wurde die Temperatur mit Hilfe des zuvor bestimmten Verschiebungskoeffizienten in Wellenlänge umgerechnet. Es lässt sich eine sehr gute Übereinstimmung der beiden Scans

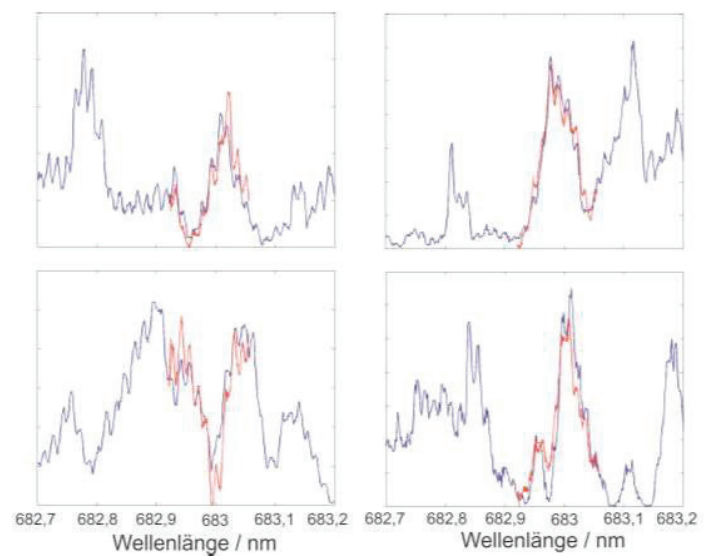

Abb. 8: Vergleich von Wellenscan (blau) mit Temperaturscan (rot) 
beobachten.

Tab.2: Übersicht über die verwendeten Kochsalzkonzentrationen und deren Brechungsindizes

\begin{tabular}{c|l|c}
$\begin{array}{c}\text { Konzentrations- } \\
\text { Nr. }\end{array}$ & \multicolumn{1}{|c|}{$\mathrm{n}$} & $\begin{array}{c}\text { Mischungsverhältnis } \\
\text { zwischen Wasser } \\
\text { und Kochsalzlösung }\end{array}$ \\
\hline 1 & 1,333 & $40: 1$ \\
2 & 1,334 & $40: 2$ \\
3 & 1,3345 & $40: 3$ \\
4 & 1,333 & $40: 1$ \\
5 & 1,3325 & $40: 0,5$ \\
6 & 1,3325 & $40: 0,25$
\end{tabular}

\section{Bestimmung des Brechungsindex}

Der Phasensprung $\delta_{\text {refl. }}$ in GI. (1) bei Totalreflexion an der Grenzfläche des Partikels ist neben dem Brechungsindex des Partikels auch vom Brechungsindex der Umgebung abhängig. Somit lässt sich der Mikropartikelarray zur Bestimmung des Brechungsindex des Umgebungsmediums verwenden. Aufgrund der geringeren Temperaturabhängigkeit und der o.g. negativen Eigenschaften von PMMA wurden auf Glaspartikel zurückgegriffen. Als Medium wurden Kochsalzlösungen unterschiedlicher Konzentration verwendet. Zunächst wurde eine gesättigte Lösung erstellt. Die Sättungskonzentration $c_{\text {sätt. }}=357 \mathrm{~g} / \mathrm{l}$ bei $25^{\circ} \mathrm{C}$ [14]) ist nur schwach temperaturabhängig und kann daher auch bei einer längeren Messzeit als konstant angenommen werden. Die Sättigungskonzentration wurde in unterschiedlichen Verhältnissen mit Wasser entsprechend Tab.2 verdünnt. Der Brechungsindex wurde mit Hilfe eine Abbe-Refraktometers (Genauigkeit $5 \cdot 10^{-4}$ ) bestimmt. In Abb. 10 ist das Ergeb-

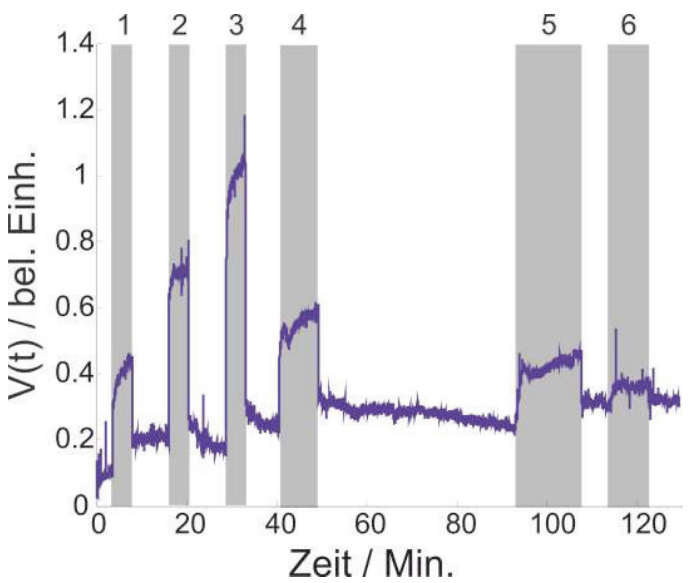

Abb. 11: Vergleichsfunktion bei Zugabe unterschiedlicher Kochsalzkonzentrationen (entsprechend

Tab.2). Zwischen den Zugaben wurde mit In/acoar rocnïlt nis einer Messung dargestellt, bei der die in Tab. 2 beschriebenen Kochsalzkonzentrationen dem Sensorarray aus Glaskugeln zugegeben wurden. Zwischen jeder Konzentrationsänderung wurde mit Wasser gespült. Es ist eine deutliche Änderung der Vergleichsfunktion erkennbar. Insbesondere zeigen sich auch Unterschiede bei den Konzentrationen 5 und 6, obwohl mit Hilfe des Refraktometers keine Unterschiede im Brechungsindex mehr messbar waren. Daher liegt die Empfindlichkeit des Mikropartikelarrays offensichtlich über der des verwendeten Refraktometers.

\section{Mikrotropfenarray als Drucksensor}

Wirken Kräfte unsymmetrisch auf einen optischen Resonator, so kann es zu einer Deformation kommen. Dies führt ebenfalls zu einer Veränderung des Resonanzverhaltens. Um eine möglichst hohe Empfindlichkeit zu erreichen müssen leicht deformierbaren Resonatoren verwendet werden. Eine Möglichkeit bieten Flüssigkeitstropfen, die in eine Silikonmatrix eingebettet sind. Der entsprechende Versuchsaufbau ist in Abb. 11 skizziert. Die Wahl der Materialien orientierte sich an der Arbeit

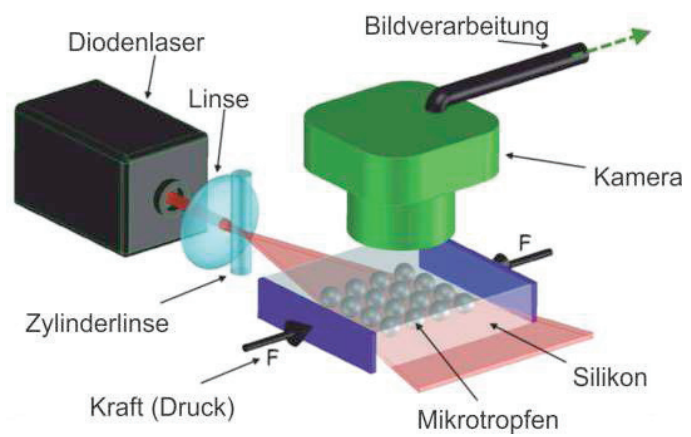

Abb. 10: Experimenteller Aufbau zur Messung von Drücken mit Hilfe eines Tropfenarrays, welcher in einer Silikonmatrix eingebettet ist.

von Saito et al. [15]. Als Trägermaterial wird das Silikon KE-103 der Fa. Shin-Etsu verwendet, das sich durch einen vergleichsweise niedrigen Brechungsindex von 1,403 und eine hohe Transparenz auszeichnet. Für die Tropfen wurde das Tensid Polysorbat 20 (Handelsname: Tween 20) mit einem Brechungsindex von 1,468 eingesetzt, welches aufgrund seiner teilweisen hydrophilen Eigenschaften in dem hydrophoben Silikon. runde Tropfen ausbildet. Anders als im Falle der zuvor verwendeten PMMA- bzw. Glaspartikel wurden die Tropfen mit einem elliptisch aufgeweiteten Freistrahl beleuchtet. Wie zuvor erfolgte die Detektion über eine CMOS-Kamera. Wie in Abb. 12 zu 


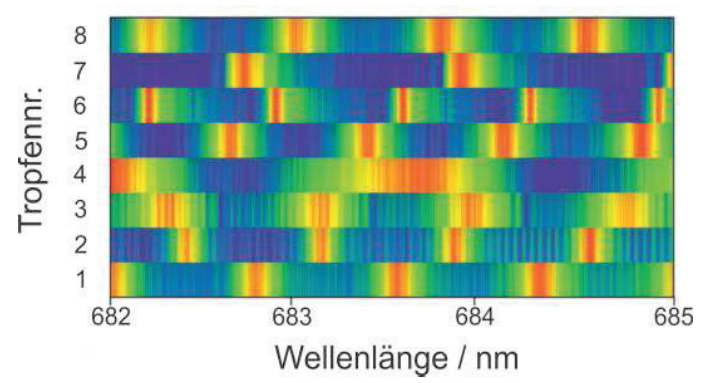

Abb. 13: Resonanzspektren von 8 gleichzeitig beleuchteten Tropfen

sehen ist, zeigten acht gleichzeitig beleuchtete Tropfen Resonanzen. Die Veränderung des Resonanzspektrums eines Tropfens aufgrund einer Druckänderung ist in Abb. 13 dargestellt. Der Druck wurde über eine Mikrometerschraube auf den Silikonblock übertragen. Eine Auswertung der Spektren ergibt eine lineare Verschiebung von $7,7 \mathrm{pm} / \mu \mathrm{m}$ (Bestimmtheitsmaß $R^{2}>99,9 \%$ ). Diese Untersuchungen zeigen, dass eine Messung mit einem solchen Tropfenarray prinzipiell möglich ist. Die direkte Anregung durch einen Freistrahl erwies sich als schwierig, da ein großer Teil des Lichts an den Partikeln gestreut wird. Das Streulicht überlagert zumeist die resonante Strahlung. Eine Möglichkeit dieses Problem zu umgehen wäre die Verwendung eines fluoreszierenden Farbstoffs im Tropfen, welcher durch die einfallende Strahlung angeregt wird. Die vom Farbstoff ausgehende Strahlung ist wellenlängenverschoben und kommt nur aus dem Inneren der Tropfen. Befindet sich ein Tropfen in Resonanz, dann wird das Fluoreszenzsignal angehoben. Es sollte sich dadurch ein deutlich erhöhter Kontrast zwischen Resonanz und Nichtresonanz ergeben.

\section{Zusammenfassung und Ausblick}

Es wurde gezeigt, dass mit einem Array aus optischen Mikroresonatoren ein vielseitiger

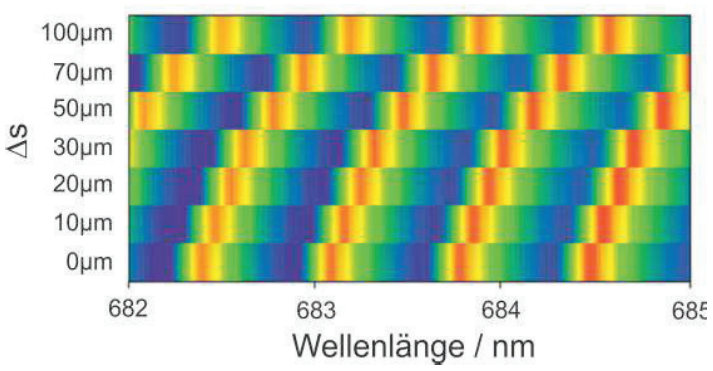

Abb. 12: Verschiebung der Resonanzen eines Tropfens aufgrund von Druckänderung. Der Druck wurde mittels einer Mikrometerschraube auf das Messvolumen übertragen (As: Einstellung der Mikrometerschraube) einsetzbarer optischer Mikrosensor bereitgestellt werden kann. Der Einsatzt eines solchen Mikroarrays zur Messungen der Wellenlänge, des Druckes, der Temperatur und der Konzentration wurde bereits nachgewiesen. Durch Funktionalisierung der Resonatoren könnte auch ein breites Einsatzgebiet in der Biologie und Medizintechnik erschlossen werden.

\section{Danksagung}

An dieser Stelle wollen wir uns für die finanzielle Unterstützung durch die Deutsche Forschungsgemeinschaft (DFG) bedanken, welche die Entwicklung dieses Sensorprinzips erst ermöglichte (GZ: OS 188/32-1, ES 182/3-2, SCHW 184/48-1).

\section{Literatur}

[1] E. Udd, W.B. Spillman (2011), Fiber Optic Sensors. 2nd ed. Chicester: Wiley.

[2] T. Yoshie, L. Tang, S.-Y. Su, Optical Microcavity: Sensing down to Single Molecules and Atoms, Sensors 7 11, S. 1972-1991 (2011).

[3] S.A. Frank Vollmer, Whispering-gallerymode biosensing: label-free detection down to single molecules, Nature Methods 5, S. 591596 (2008).

[4] A.M. Armani, R.P. Kulkarni, S.E. Fraser, R.C. Flagan, K.J. Vahala, Label-Free, SingleMolecule Detection with Optical Microcavities, Science 317, S. 783-787 (2007).

[5] H.C.d. van Hulst (1981), Light scattering by small particles. Unabridged and corr. republ. of the work orig. publ. in 1957, New York. New York, NY: Dover.

[6] G. Roll, G. Schweiger, Geometrical optics model of Mie resonances, J. Opt. Soc. Am. B 17, S. 1301-1311 (2000).

[7] G. Schweiger, M. Horn, Effect of changes in size and index of refraction on the resonance wavelength of microspheres, J. Opt. Soc. Am. B 23, S. 212-217 (2006).

[8] R. Watson, M. Yi, Thermal expansion and hydration behavior of PMMA molding materials for LIGA applications, Sand report 2003-8000 (2003).

[9] S. Ledesma, S.N. Goyanes, C. Duplaá, Development of a dilatometer based on diffractometry, Rev. Sci. Instrum. 73, S. 32713274 (2002).

[10] P. Michel, J. Dugas, J. Cariou, L. Martin, Thermal variations of refractive index of PMMA, polystyrene, and poly (4- methyl-1 - 
pentene), J. Macromol. Sci.-Phys., B 25, S. 379-394 (1986).

[11] Schott AG (2009), Optisches Glas - Beschreibung der Eigenschaften 2009.

[12] D. Leviton, B. Frey, Temperaturedependent absolute refractive index measurements of synthetic fused silica, ArXiv e-prints 0805.0091 (2008).

[13] J. Lehmann, Wasseraufnahme von PMMA und $P C$, KU Prüftechnik 91, S. 80-83 (2001).

[14] Sigma Aldrich Inc., Product Information: Sodium Chloride.

[15] M. Saito, H. Shimatani, H. Naruhashi, Tunable whispering gallery mode emission from a microdroplet in elastomer, Opt. Express 16, S. 11915-11919 (2008). 